Thomas Endres · René Grass · Achim Biewener · Till Illert · Hans Zwipp

Klinik und Poliklinik für Unfall- und Wiederherstellungschirurgie, Technische Universität Dresden

\title{
Minimal traumatisierende Versorgung der Pilon-tibiale-Frakturen
}

\section{Schlüsselwörter}

Ilizarov $\cdot$ llizarov-Hybrid-Fixateur .

Pilonfraktur · Hochrasanztrauma ·

Minimalinvasive Techniken versorgten 62 Pilonfrakturen bei 59 Patienten konnten in einer retrospektiven Untersuchung 50 Frakturen bei 49 Patienten nachuntersucht werden (81\%). In Gruppe I (llizarov-Technik kombiniert mit minimalinvasiver interner Osteosynthese, $n=11$ ) kamen 43-C2/C3-Frakturen (73\%) sowie schwer wiegende Weichteilschäden und insbesondere offene Verletzungen (100\%) signifikant häufiger vor als in Gruppe II (konventionelles Operationsverfahren, $n=38$ ) mit $33,3 \%$ bzw. $38 \%$. Dennoch wurden in Gruppe I im Verlauf keine Pseudarthrosen oder Osteitiden beobachtet und im Langzeitverlauf keine Arthrodese notwendig. In Gruppe II dagegen wurden in $5 \%$ eine 0steitis, in 2,5\% eine verzögerte Knochenbruchheilung konstatiert und in $8 \%$ eine Arthrodese notwendig. Nachteilig waren die in Gruppe I relativ häufig auftretenden Pininfekte (45\%) mit einem revisionspflichtigem Befund in 18\%. Bei isolierter Auswertung der C2-, C3-Frakturen erreichten über $87 \%$ der Patienten aus Gruppe I und nur 38\% der Patienten aus Gruppe II ein sehr gutes oder gutes Maryland-Foot-ScoreErgebnis. Die mit einer minimalinvasiven internen Osteosynthese kombinierte llizarovTechnik ist somit ein effizientes Verfahren zur Therapie von komplexen Pilon-tibialeFrakturen mit schwerem Weichteilschaden. on Jergesen [14] wurden im Jahr 1959 die offene Reposition und Stabilisierung von Pilon-tibiale-Frakturen noch für unmöglich angesehen. Bereits 10 Jahre später berichteten Ruedi u. Allgöwer [27] jedoch erstmalig über die Ergebnisse von 84 operativ behandelten Pilon-tibiale-Frakturen. Sie inaugurierten eine 4-schrittige Operationsstrategie, die bis heute die Grundlage der operativen Therapie von Pilon-tibiale-Frakturen darstellt $[27,28]$. In ihrem Patientengut mit einem hohen Anteil (75\%) an Sportverletzungen (low energy trauma) sahen sie in 2/3 der Fälle operativ versorgter Pilon-tibiale-Frakturen sehr gute bis gute Ergebnisse [27, 28].

In den letzten 2 Jahrzehnten wurde insgesamt über weniger vorteilhafte Ergebnisse und eine alarmierende Zunahme der Komplikationsinzidenz nach offener Reposition und Plattenosteosynthese berichtet $[5,8,9,10,13,23,35]$. Eine akzeptierte Erklärung hierfür ist, dass anders als in den Patientenkollektiven von Ruedi u. Allgöwer [27, 28] - heute $60-70 \%$ der Pilon-tibiale-Frakturen auf ein Hochrasanztrauma (high energy trauma) durch Sturz aus großer Höhe oder Verkehrsunfälle zurückgehen [12, $19,21,33]$. Hochrasanzverletzungen des Pilon tibiale sind gekennzeichnet durch

- eine komplexe Frakturmorphologie und

- einen schweren Weichteilschaden $[5,12,19,26]$.

Da die Frakturheilung maßgeblich von der Vitalität des ohnehin problematischen Weichteilmantels im Bereich der distalen Tibia und dem Erhalt der Vaskularität im Bereich der Frakturzone abhängig ist, wird mit Zunahme des traumatischen und auch operationstechnisch bedingten Weichteilschadens die Frakturheilung entsprechend negativ beeinflusst $[9,19]$. Es überrascht daher nicht, dass mit Zunahme des traumatischen Weichteilschadens und der zusätzlichen operativ bedingten Weichteildissektion nachweislich die perioperative Komplikations-, Osteitis- und Pseudarthroseninzidenz ansteigen, schwere Arthroseformen häufiger beobachtet werden und das Endergebnis nachhaltig negativ beeinflusst wird $[12,19,20,21,26$,

\footnotetext{
(c) Springer-Verlag 2003

Dr.Thomas Endres

Klinik und Poliklinik für Unfallund Wiederherstellungschirurgie, Technische Universität, TU-Dresden, Fetscherstraße 74,01307 Dresden, E-Mail:ThEnd64@aol.com, Phone: 0351-4582761, Fax: 0351-4584379
} 
T. Endres $\cdot$ R. Grass $\cdot$ A. Biewener $\cdot$ T. Illert · H.Zwipp

\section{Minimally invasive treatment of pilon fractures of the tibia}

\section{Abstract High-energy-trauma · Pilon . Minimalinvasive technics}

Between October 1993 and September 1999 a total of 62 pilon-tibiale fractures in 59 patients were treated at the Clinic for Trauma and Reconstructive Surgery, University Hospital Carl-Gustav-Carus, Technical University Dresden. In a retrospective study 49 patients with 50 pilon-tibiale fractures $(81 \%)$ could be examined clinically and radiographically an average after 28 months after injury. The purpose of this study was to compare the healing results clinically and radiographically by using the llizarov-technique in combination with a minimalinvasive, internal fixation (group I) with those by using a conventional surgical procedure (internal fixation with a plate, external fixation with or without minimalinvasive, internal fixation and screw fixation exclusively / group II) and to evaluate the significance of the llizarov-technique. Data analysis showed a significantly increased incidence of 43-C2/C3-fractures in the llizarov-group I $(73 \%)$ than in group II (33.3\%). Severe soft tissue injuries and particularly open injuries had a significantly increased incidence in the Ilizarov-group I (100\%) than in the group II (38\%). Despite of the high incidence of C2-, C3-fractures and severe soft tissue injuries in the group I there was no incidence of pseudoarthrosis or osteitis in the further course and there was no need of an arthrodesis during the long-term course. After therapy with a conventional surgical technique the incidence of an osteitis was $5 \%$ and of a delayed union of fracture $2.5 \%$ and an arthrodesis was necessary at $8 \%$. A disadvantage of the llizarov-system was the relatively frequent incidence of pin-infection (45\%) with the necessity of surgical débridement at $18 \%$. Correspondingly the surgical outcome of $82 \%$ of group I-patients and only $51 \%$ of group IIpatients was rated very well or well. The efficiancy of the treatment of 43-C2-, C3-fractures with the llizarov-technique was obvious by a statistically, significantly better Maryland-Foot-Score in comparison with the group II. More than $87 \%$ of the patients treated with the llizarov-technique and only $38 \%$ of the patients treated with a conventional surgical procedure obtained a very good or good score. According to these findings the Ilizarov-technique in combination with a minimal-invasive, internal fixation is an effec- tive method to treat complicated pilon-tibiale fractures with a severe soft tissue trauma.

\section{Keywords}

Ilizarov method $\cdot$ Hybrid external fixator ·

35]. Eine erhöhte Infektions- und Arthroserate nach offener Reposition und Plattenosteosynthese dürften folglich bei Hochrasanztraumen $[4,6,10,11$, $13,19,23,24,35]$ auf die, zusätzlich zu dem initial bestehenden traumatischen Weichteilschaden, operativ bedingte Weichteildissektion zurückzuführen sein. Die beobachteten

- Komplikationsraten von bis zu 70\% [35],

- schweren Arthroseformen in bis zu $43 \%$ [19],

- Osteitisraten von bis zu 33\% [9],

- Pseudarthroseninzidenz bis zu $27 \%$ [35] und

- Arthrodesenraten bis zu 32\% [8]

sind vermeidbar, wenn der zentralen Bedeutung des Weichteilschadens Rechnung getragen wird und die operativ bedingte Weichteildissektion, als eine durch den Unfallchirurgen beeinflussbare Determinante, durch Konzentration auf minimalinvasive Techniken minimiert wird.

\section{Grundprinzipien der operativen Versorgung von Pilon-tibiale-Frakturen}

Die Versorgung von Hochrasanztraumen des Pilon tibiale erfordert ein mehrzeitiges Vorgehen. Eine einzeitige Vorgehensweise birgt nachweislich die Gefahr eines erhöhten Infektionsrisikos [4, 21, 26, 33].

Primär muss die Wiederherstellung des lateralen Pfeilers durch Rekonstruktion der Fibula in Länge, Achse und Rotation erfolgen, um eine korrekte Lagebeziehung zum Talus zu erreichen. $\mathrm{Zu}$ sätzlich ist in der Regel eine temporäre OSG-Transfixation mit einem konventionellen Fixateur externe zur Konsolidierung der Weichteile erforderlich.

Bei zweit- bis drittgradig offenen und drittgradig geschlossenen Verlet- zungen werden vor der definitiven Versorgung in der Regel mehrere Weichteildébridements notwendig.

Erst nach Konsolidierung der Weichteile sind sekundär die stufenlose, anatomische Rekonstruktion des Tibiaplafonds und anschließend die stabile Retention der meta- bzw. diaphysären Fakturzone durch verschiedene Techniken möglich.

\section{Techniken der minimalinvasiven stufenlosen Rekonstruktion des Tibiaplafonds}

Zur minimalinvasiven stufenlosen Rekonstruktion des Tibiaplafonds stehen verschiedene Techniken zur Auswahl. Die Indikation zur jeweiligen Technik ist vom Frakturtyp abhängig.

\section{Geschlossene Reposition mit perkutaner Schraubenosteosynthese}

Spaltbrüche, wie B1.1-, B1.2-, B1.3-, C1.1, C1.2- und C1.3-Frakturen, können in der Regel mit Hilfe von Joysticks (KirschnerDrähte, Schanz-Schrauben) und einer perkutan eingebrachten Repositionszange geschlossen reponiert und anschließend durch perkutane Schraubenosteosynthese retiniert werden (Abb. 1).

\section{Arthroskopisch gestützte Reposition}

Eine arthroskopisch gestützte Reposition gelingt in der Regel bei den weniger komplexen Gelenkfrakturen mit Impression der Gelenkfläche wie B2.1-, B2.2-, B2.3-Frakturen und ggf. auch C2.1und C2.2-Frakturen.

Unter arthroskopischer Sicht wird die Impression antegrad über ein Kortikalisfenster aufgestößelt. Anschließend wird die Fraktur wie unter Punkt 1 beschrieben durch eine perkutane Schraubenosteosynthese retiniert (Abb. 2).

\section{Limitiert offene Reposition}

Eine limitiert offene Reposition über einen kleinen, strikt medianen Zugang ist bei allen komplexeren Gelenkfrakturen, wie B3-, C2- und C3-Frakturen, notwendig. 


\section{Unterschenkel- und obere Sprunggelenksfrakturen}
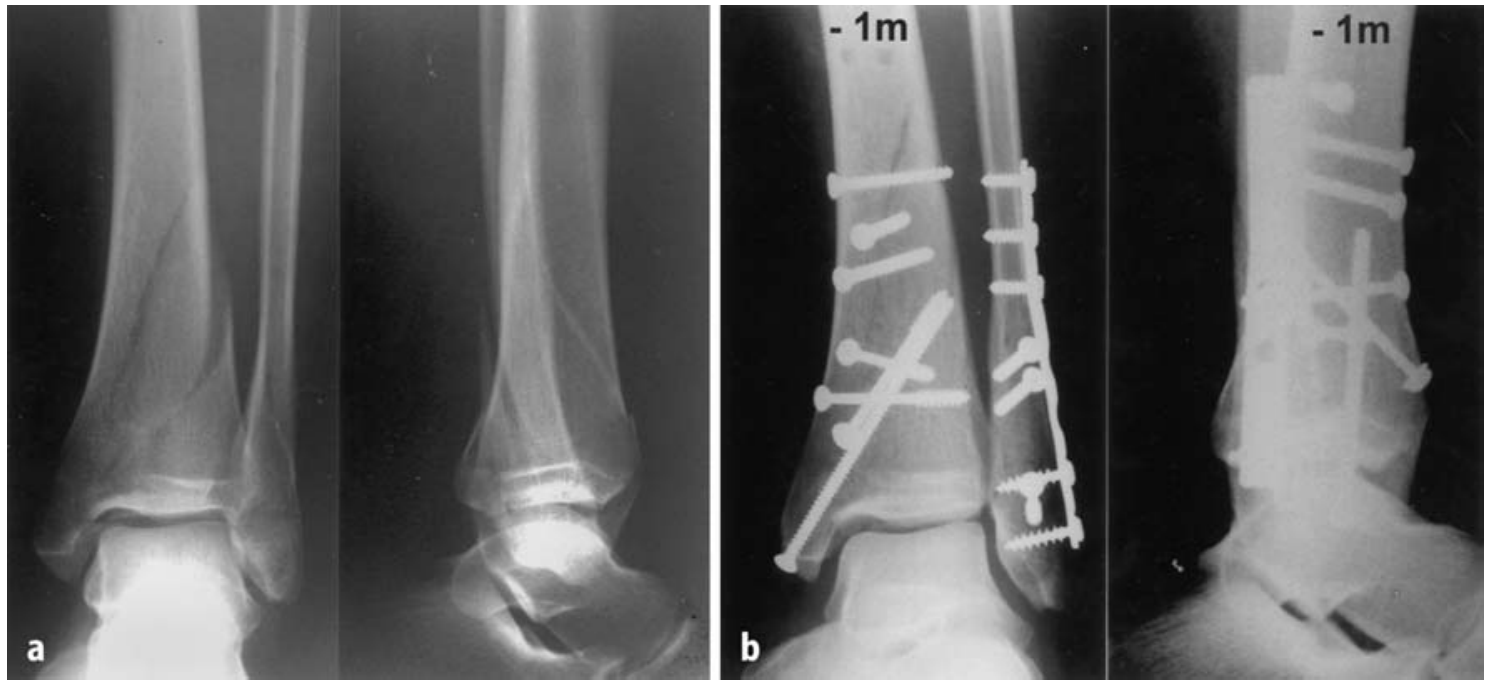

Abb. 1a,b $\Delta$ Beispiel einer minimalinvasiven Versorgung einer C1.3-Fraktur (a) durch geschlossene Reposition und Retention durch perkutane Schraubenosteosynthese (b)

\section{Techniken der minimalinvasiven stabilen Retention der meta- und diaphysären Frakturzone}

Nach der anatomischen Rekonstruktion des Gelenkblocks ist eine suffiziente stabile Retention der meta- und diaphysären Frakturausläufer, insbesondere bei 43-C2/C3-Frakturen, zur Vermeidung einer Varusfehlstellung notwendig. Dies gelingt in der Regel mit einer medial eingeschobenen Plattenosteosynthese oder durch eine Ilizarov-Montage.

\section{Eingeschobene Plattenosteosynthese (LC-DCP)}

Über eine mediale Inzision wird die Platte durch eine perkutane Tunnelierungstechnik subkutan nach proximal hochgeschoben, die Plattenlage wird über eine weitere Inzision am proximalen Plattenende kontrolliert. Anschließend erfolgt die perkutane Schraubenbesetzung der Platte unter Bildwandlerkontrolle.

\section{Ilizarov-3-Vollring-Montage, Ilizarov-Hybrid-Konstruktion}

Die Anwendung der Ilizarov-Technik mit ihrer kaum zu überbietenden Gewebeschonung, einer Vielzahl von primären und sekundären Repositionsmöglichkeiten und einer hohen primären Belastungsfähigkeit wurde in der Literatur vereinzelt für die Therapie von $\mathrm{Pi}$ lon-tibiale-Frakturen beschrieben $[1,2$, $16,17,18,22,36]$. Das Ilizarov-System eig- net sich besonders für die Therapie von Pilon-tibiale-Frakturen mit einer ausgedehnten metaphysären Trümmerzone, wie 43-C2/C3-Frakturen (Abb. 3). Durch die hohe primäre Belastungsfähigkeit des Ilizarov-Systems kann bei Pilon-tibiale-Frakturen mit komplexer metaphysärer Trümmerzone auf eine mediale Abstützung durch Plattenosteosynthese verzichtet und dadurch die prekäre metaphysäre Fragmentdurchblutung geschont werden. Zugangsbedingte Weichteildissektionen werden im Gegensatz zur Plattenosteosynthese durch das Ilizarov-System vermieden. Somit kann das Implantat als biologische Osteosynthese charakterisiert werden.

Die dynamische Stabilität des Systems mit seinem nichtlinearen, axialen Steifigkeitsverhalten lässt, im Gegensatz $\mathrm{zu}$ rigiden konventionellen Fixateur-externe-Verfahren oder einer statischen Plattenosteosynthese, bei geringen axialen Belastungen dosierte axiale Bewegungsauslenkungen zu und fördert dadurch die reparativen Prozesse der Frakturheilung, während das Steifigkeitsverhalten unter zunehmender Belastung ansteigt und ein Schutz der reparativen Knochenprozesse vor Gewebeüberdehnung bzw. zu großer Bewegungsauslenkung im Frakturspalt gewährleistet wird $[15,30]$.

\section{Eigenes Patientengut}

Im Zeitraum von Oktober 1993-September 1999 wurden 62 Pilon-tibiale-Frak- turen bei 59 Patienten operativ versorgt. Patienten mit Sekundärversorgung, bei bereits auswärtig erfolgter primärer operativer Therapie, wurden von der Studie ausgeschlossen. In einer retrospektiven Analyse konnten 49 Patienten mit 50 Pilon-tibiale-Frakturen (Followup-Rate $81 \%$ ) durchschnittlich nach 28 Monaten klinisch und radiologisch nachuntersucht werden.

Zur Evaluierung der Effizienz der Ilizarov-Technik in Kombination mit einer arthroskopisch gestützten oder limitiert offenen Reposition und Retention der Tibiaplafondfragmente durch Schraubenosteosynthese im Vergleich zu konventionellen Operationsverfahren bei Pilon-tibiale-Frakturen wurde eine Einteilung in 2 Gruppen vorgenommen:

\section{Gruppe I}

11 Patienten mit einem relevanten Weichteilschaden und komplexer Frakturmorphologie wurden mit der IlizarovTechnik, 6-mal als Hybridkonstruktion und 5-mal als 3-Vollring-Montage, behandelt. Dabei erfolgte, nach Wiederherstellung der Länge und Osteosynthese der Fibula, zunächst eine minimalinvasive, anatomische Rekonstruktion der tibialen Gelenkfläche über einen kleinen, strikt medianen Zugang in 9 Fällen oder arthroskopisch gestützt perkutan in 2 Fällen durch Schraubenosteosynthese (KFI).

In einem 2. Operationsschritt wurde die notwendige Retention der meta- und 


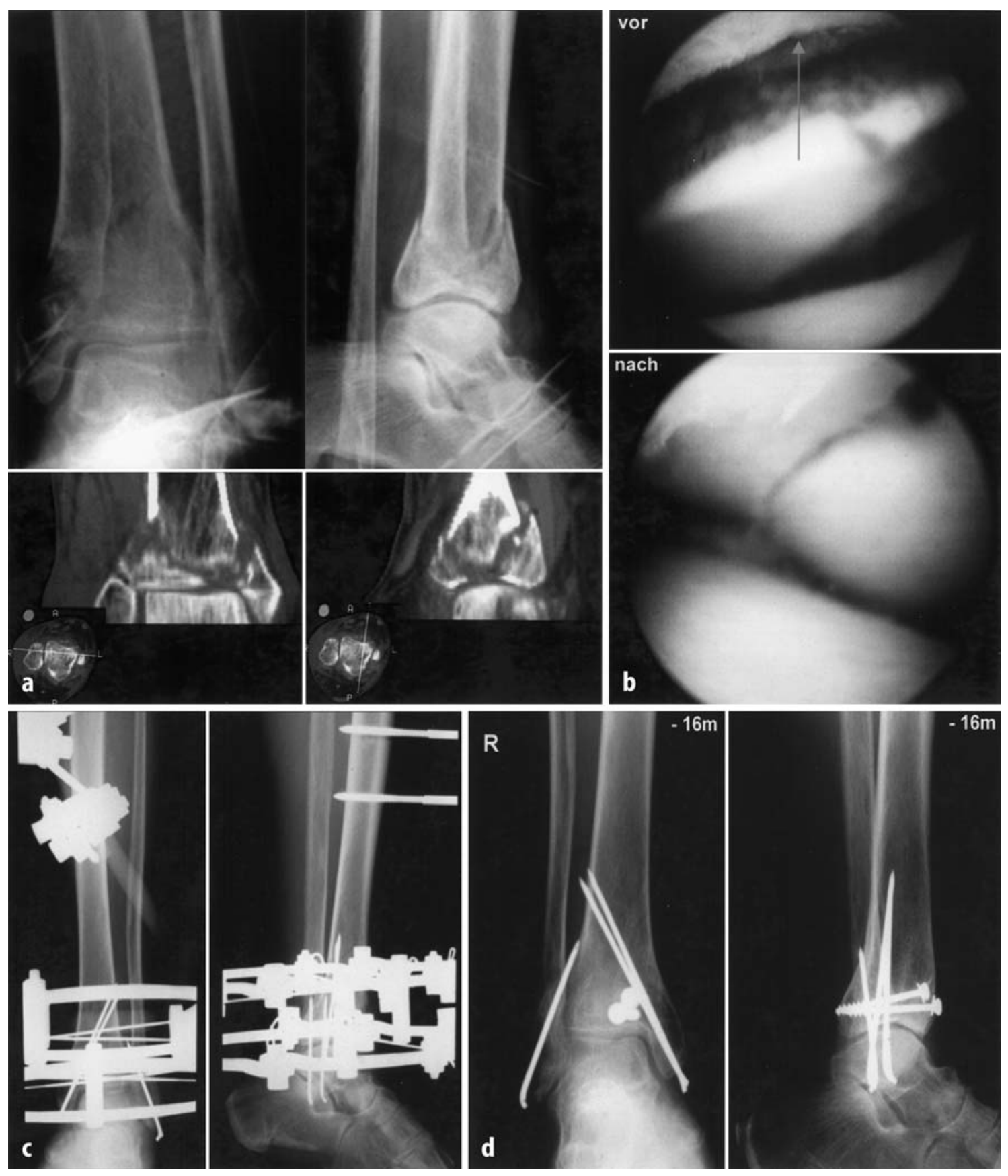

Abb. 2a-d $\Delta$ Beispiel einer arthroskopisch gestützten Reposition einer C2.1-Fraktur mit zweitgradigem geschlossenem Weichteilschaden (a). In der Arthroskopie deutlich sichtbare Stufenbildung und nach arthroskopisch gestützter Reposition und Retention mit Schrauben- und Kirschner-Drähten sichtbares stufenloses Repositionsergebnis (b). Retention der metaphysären Fraktur durch eine llizarov-Hybrid-Konstruktion (c). Ausheilungsergebnis nach 16 Monaten (d)

diaphysären (Trümmer-)Fraktur durch das Ilizarov-System erreicht (Abb. 2, 3).

Die Ilizarov-Konstruktion war in 9 Fällen nicht gelenküberbrückend und in 2 Fällen temporär für durchschnittlich 16,4 Tage gelenküberbrückend.

Eine Vollbelastung wurde - je nach vorliegender Frakturmorphologie - in der Regel nach 6-8 Wochen gestattet und durchschnittlich nach insgesamt 11 Wochen (6-18 Wochen) erreicht. Der Ilizarov-Fixateur wurde durchschnittlich nach 12 Wochen entfernt.

\section{Gruppe II}

In Gruppe II waren 38 Patienten mit 39 Pilonfrakturen durch konventionelle
Operationsverfahren behandelt worden. 8 Patienten waren mit Plattenosteosynthese, 2-mal primär und 6-mal sekundär, versorgt worden. In 31 Fällen war eine minimalinvasive Osteosynthese (MIO), 12-mal in Form einer Osteosynthesekombination aus konventionellem Fixateur externe und Schraubenosteosynthese, 3-mal in Form einer alleinigen 


\section{Unterschenkel- und obere Sprunggelenksfrakturen}
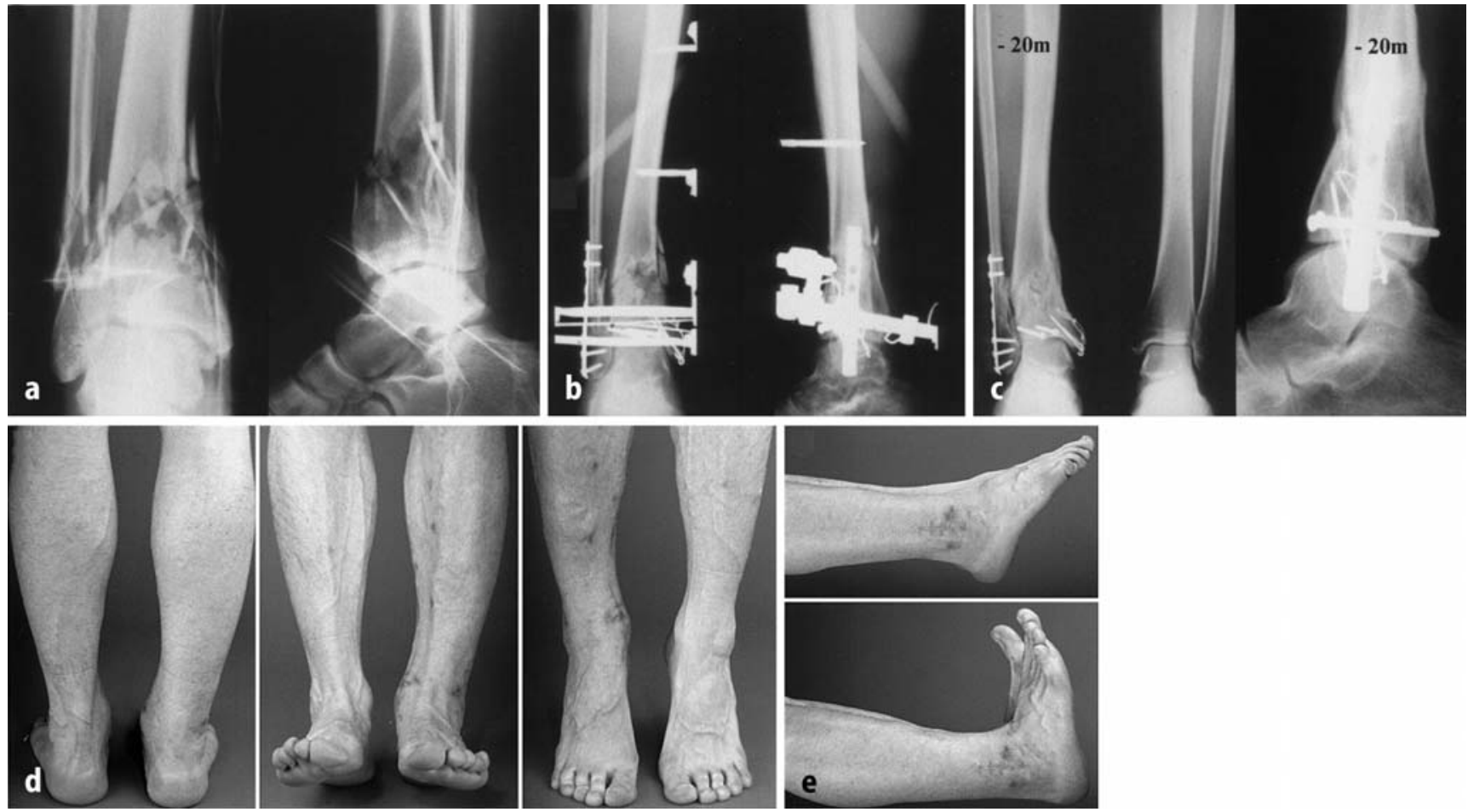

Abb. 3a-e $\Delta$ 31-jähriger Patient mit einer erstgradigen offenen C2.2-Fraktur des Pilon tibiale nach Hochrasanztrauma (Motorradunfall). a Unfallbilder, b Rekonstruktion des Gelenkblocks über eine limitiert offene Reposition und Retention durch Schraubenosteosynthese, Retention der diaphysären Trümmerzone durch eine Ilizarov-Hybrid-Konstruktion, c-e Ausheilungsergebnis nach 20 Monaten (c) mit einem guten funktionellem Ergebnis ( $d, e)$ (Maryland-Foot-Score: 97 Punkte) und völliger Beschwerdefreiheit

Anlage eines AO-Fixateur externe und 16-mal in Form einer alleinigen Schraubenosteosynthese durchgeführt worden.

Eine Vollbelastung wurde in der Regel bei einfacher Frakturmorphologie nach 6 Wochen, bei komplexer Frakturmorphologie nach 8-12 Wochen gestattet und nach durchschnittlich 13 Wochen (6-27 Wochen) erreicht.

\section{Ergebnisse}

\section{Demographische Daten}

Die häufigste Unfallursache im Gesamtkollektiv war der Sturz aus großer Höhe mit $46 \%$, gefolgt von Verkehrsunfällen mit 30\%. Dabei betrug der Anteil an Hochrasanztraumen in Gruppe I $64 \%$ und in Gruppe II 59\% (Tabelle 1).

\begin{tabular}{|c|c|c|c|}
\hline Demographie & $\begin{array}{l}\text { Gruppe I } \\
\text { (Ilizarov) [\%] }\end{array}$ & $\begin{array}{l}\text { Gruppe II } \\
\text { (konventionell) [\%] }\end{array}$ & Statistik \\
\hline Hochrasanztrauma & 64 & 59 & n. s. \\
\hline Polytraumatisierung & 0 & 10 & n. s. \\
\hline Mehrfach Verletzte & 18 & 51 & $p=0,013$ \\
\hline $\begin{array}{l}\text { Konkomitierende Talus- bzw. Kalkaneusfrakur } \\
\text { Ipsilateral } \\
\text { Kontralateral }\end{array}$ & - & $\begin{array}{r}8 \\
21\end{array}$ & $p=0,045$ \\
\hline $\begin{array}{l}\text { Relevanter Vorschaden (Arthrose, Zustand nach } \\
\text { OSG-Fraktur, Fehlstellung) }\end{array}$ & 46 & 5 & $p=0,004$ \\
\hline Relevante Vorerkrankungen & 36 & 10 & n. s. \\
\hline
\end{tabular}

Ein statistisch signifikanter Unterschied zwischen beiden Gruppen bestand hinsichtlich der Verteilung von mehrfach verletzten und polytraumatisierten Patienten $(p=0,013)$.Während in Gruppe I keine Patienten polytraumatisiert und nur $18 \%$ der Patienten mehrfach verletzt waren, waren in Gruppe II $10 \%(n=4)$ der Patienten polytraumatisiert und 51\% $(n=20)$ der Patienten mehrfach verletzt (Tabelle 1).

Demgegenüber lag in Gruppe I ein relevanter Vorschaden in Form einer vorbestehenden Arthrose des OSG, einer älteren fehlverheilten OSG-Fraktur bzw. einer schweren, ipsilateralen Fußfehlstellung mit Veränderung der Rückfußgeometrie in $46 \%(n=5)$ statistisch signifikant häufiger als in Gruppe II mit $5 \%$ $(n=2)$ vor $(p=0,004)$.

Relevante Vorerkrankungen mit möglicher negativer Beeinflussung des Ausheilungsergebnisses wie z. B. pAVK, insulinpflichtiger Diabetes mellitus, chronisch venöses Ulcus cruris sowie erheblicher Alkoholabusus fanden sich mit $36,4 \%(n=4)$ in Gruppe I wesentlich häufiger als in Gruppe II mit $10 \%(n=4)(p=0,059)$. 


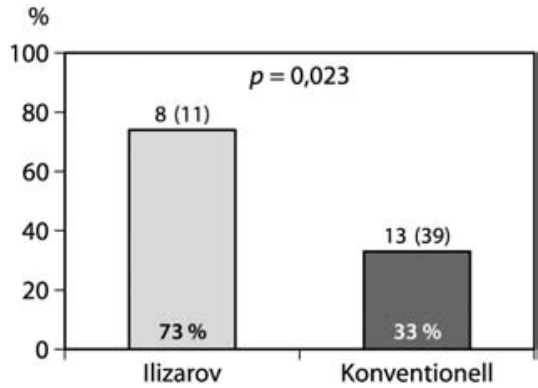

Abb. $4 \Delta$ Häufigkeit von C2- und C3-Frakturen, in der Ilizarov-Gruppe mit $73 \%$ signifikant häufiger als in Gruppe II mit 33\% $(p=0,023)$

\section{Frakturmorphologie/ Weichteilschaden}

In Gruppe I traten ausschließlich C-Verletzungen und in Gruppe II $\mathrm{zu} 54 \%$ $\mathrm{B}$-Verletzungen und $\mathrm{zu} 46 \% \mathrm{C}$-Verletzungen auf. Schwierig zu versorgende komplexe Frakturmorphologien, entsprechend 43-C2/C3-Frakturen, waren in Gruppe I $[72,7 \%(n=8)]$ statistisch signifikant häufiger vertreten als in Gruppe II $[33,3 \%(n=13)](p=0,023)($ Abb. 4$)$. In Gruppe II dominierten mit $61,5 \%$ die relativ unproblematisch zu versorgenden 43-B1-, -B2- und -C1-Frakturen.

Ein statistisch signifikanter Gruppenunterschied fand sich in der Häufigkeitsverteilung des Weichteilschadens. Weichteilschäden und insbesondere offene Verletzungen waren statistisch signifikant häufiger in Gruppe I als in Gruppe II vertreten $(p<0,0005 / p=0,014)$ (Abb. 5). Korrelierend mit der Schwere der Frakturmorphologie fand sich in allen Fällen der Gruppe I ein prognostisch ungünstig zu wertender, schwerer Weichteilschaden (Abb. 5). Bei 1 Patienten lag ein erstgradiger, bei 4 ein zweitgradiger und bei 1 Patienten ein drittgradiger offener Weichteilschaden vor, sodass bei insgesamt $54,5 \%(n=6)$ der Fälle der Gruppe I ein offener Weichteilschaden $\mathrm{zu}$ konstatieren war. Bei den restlichen 5 Patienten (45,5\%) bestand ein geschlossener Weichteilschaden 3. Grads.

Im Gegensatz dazu überwog in Gruppe II mit 61,5\% (n=24) der Fälle der Anteil an Patienten, die keinen oder einen $\mathrm{zu}$ vernachlässigenden Weichteilschaden aufwiesen. Nur bei 15,4\% $(n=6)$ der Patienten der Gruppe II bestand ein offener Weichteilschaden und bei $23 \%$ $(n=9)$ ein höhergradig geschlossener Weichteilschaden zweiten oder dritten Grads.

\section{Komplikationen}

Trotz des hohen Anteils an $\mathrm{C}_{2}-$ und $\mathrm{C}_{3}$ Frakturen $\left(43-\mathrm{C}_{2} / \mathrm{C}_{3}\right)$ in der mit dem Ilizarov-System versorgten Gruppe I wurden in dieser keine verzögerte Knochenbruchheilung oder eine Pseudarthrose beobachtet (Tabelle 2).

Ein Pininfekt als typische Komplikation des Ilizarov-Systems trat bei $5 \mathrm{~Pa}$ tienten der Gruppe I auf (Tabelle 2). Bei 2 dieser Patienten war eine Revision erforderlich, und bei 3 Patienten musste der Ilizarov-Fixateur nach einer durchschnittlichen Fixationsdauer von $73 \mathrm{Ta}$ gen infolge des Pininfekts vorzeitig entfernt werden. Ein Verfahrenswechsel war in keinem Fall notwendig.

In Gruppe II fand sich ein revisionspflichtiger Pininfekt in der Patientengruppe, die u. a. mit einem konventionellen Fixateur externe behandelt worden war.

Die Osteitisrate des Gesamtkollektivs (Gruppe I und II) entsprach $4 \%(n=2)$. Ei-

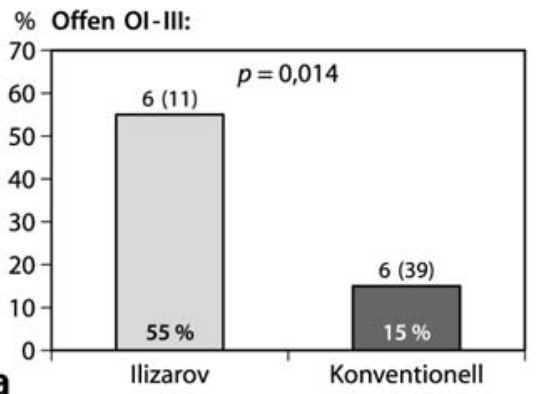

Abb. 5a,b $\Delta$ Weichteilschaden. In der Ilizarov-Gruppe waren, korrelierend mit der Frakturmorphologie, signifikant häufiger Pilon-tibiale-Frakturen mit schwerem Weichteilschaden vertreten als in Gruppe II $(p=0,0005)$, a offene, b geschlossene Frakturen

Tabelle 2

Komplikationshäufigkeit in beiden Gruppen. Außer den für die llizarov-Technik typischen, in aller Regel folgenlos ausheilenden Pininfekten traten in der Ilizarov-Gruppe trotz des hohen Anteils an 43-C2/C3-Frakturen und schweren Weichteilschäden keine schwerwiegenden Komplikationen wie Osteitis und Pseudarthrose auf

\begin{tabular}{lccl} 
Komplikationen & $\begin{array}{l}\text { Gruppe I } \\
\text { (Ilizarov) }\end{array}$ & $\begin{array}{l}\text { Gruppe II } \\
\text { (konventionell) }\end{array}$ & Statistik \\
\hline Pininfekt & $45 \%(5 / 11)$ & $13 \%(2 / 15)$ & $p=0,034$ \\
Osteitis & $0 \%(0 / 11)$ & $5 \%(2 / 39)$ & n. s. \\
Verzögerte Knochenbruchheilung & $0 \%(0 / 11)$ & $2,5 \%(1 / 39)$ & n. s. \\
Pseudarthrose & $0 \%(0 / 11)$ & $0 \%(0 / 39)$ & n. s. \\
Höhergradige Fehlstellungen über $10^{\circ}$ & $0 \%(0 / 11)$ & $10 \%(4 / 39)$ & n. s. \\
M.-peronaeus-superficialis-Läsion & $9 \%(1 / 11)$ & $5 \%(2 / 39)$ & n. s. \\
Arthrolyse & $0 \%(0 / 11)$ & $5 \%(2 / 39)$ & n. s.
\end{tabular}

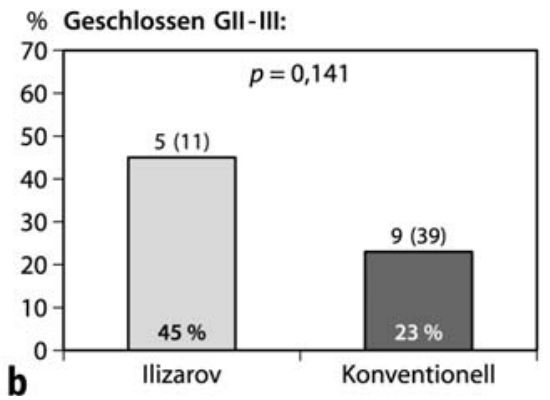

ne Osteitis wurde in der Ilizarov-Gruppe, trotz des hohen Anteils an $\mathrm{C}_{2}-$, $\mathrm{C}_{3}$-Frakuren und schwerem Weichteilschaden, in in Fall und in Gruppe II bei 2 (5\%) mit $\mathrm{C}_{2}$-Frakturen beobachtet (Tabelle 2). Ein Verfahrenswechsel war in eiden Fällen erforderlich.

bei komplexer Frakturmorpho bestehender Osteoporose auf. In der Ilizarov-Gruppe wurden Fehlstellungen von über $5-10^{\circ}$ bei $27 \%(n=3)$ der Fälle, 2mal in Form einer Varusdeformität von $6^{\circ}$ und $7^{\circ}$ sowie 1 -mal als Valgusdeformität von $9^{\circ}$, beobachtet. Höhergradige Fehlstellungen von über $10^{\circ}$ traten in der arov-Gruppe in keinem Fall auf. Alle tstellungen wurden nach AnwenFei 2 der 3 Patienten trat die nung des Ilizarov-Fixateurs wegen eines Pininfekts auf. Eine Korrekturosteotomie war bei fehlender Beschwerdesymptomatik in keinem Fall notwendig. 


\section{Unterschenkel- und obere Sprunggelenksfrakturen}

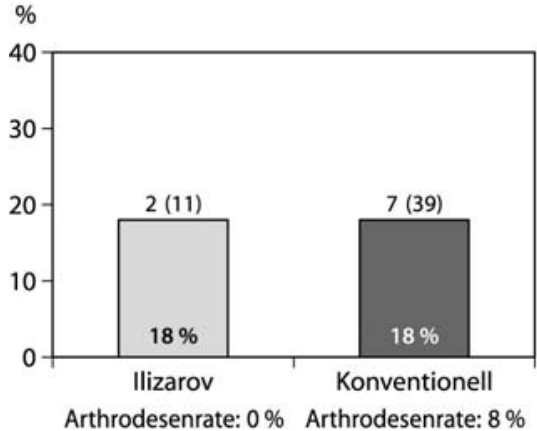

Abb. $6 \Delta$ Schwere Arthrosefomen 2.-3.Grads nach Bargon traten in beiden Gruppen zu jeweils $18 \%$ ausschließlich bei C2- und C3-Frakturen auf. Eine Arthrodese war lediglich in $8 \%$ der Fälle der Gruppe II notwendig und in keinem Fall der Gruppe I

Nach Versorgung mit konventionellen Operationsverfahren wurden in Gruppe II bei 10\% $(n=4)$ eine Fehlstellung von $5-10^{\circ}$ und im Gegensatz zur Gruppe I auch höhergradige Fehlstellungen von über $10^{\circ}$ bei $10 \%(n=4)$ beobachtet. Bei 3 der 4 Patienten mit höhergradigen Fehlstellungen erfolgte sekundär eine Korrekturosteotomie.

Ein statistisch signifikanter Unterschied bezüglich der Fehlstellungsrate zwischen beiden Gruppen konnte ebenso wenig wie eine Korrelation zwischen Fehlstellungsrate und späterer Arthrose ermittelt werden.

\section{Arthrose/Arthrodese}

Schwere Arthroseformen 2.-3. Grads nach Bargon wurden im Rahmen der Nachuntersuchung in beiden Gruppen $\mathrm{zu}$ jeweils $18 \%$ gesehen und traten ausschließlich bei $\mathrm{C}_{2}$ - und $\mathrm{C}_{3}$-Frakturen auf.

Bezogen auf das Gesamtkollektiv (Gruppe I und II) wurde eine Arthrodesenrate von $6 \%$ evaluiert. Während nach der minimalinvasiven Versorgung mit dem Ilizarov-System in Gruppe I in keinem Fall eine Arthrodese notwendig wurde, wurde bei 3 der 7 Patienten der Gruppe II mit fortgeschrittener Arthrose eine sekundäre Arthrodese des OSG durchschnittlich 14,3 Monate nach dem Unfallereignis erforderlich (Abb. 6).

Die Arthrodesenrate von $8 \%$ nach Versorgung mit konventioneller Operationstechnik unterschied sich nicht signifikant von der nach Versorgung mit der Ilizarov-Technik $(p=0,466)$.

\section{Subjektive Parameter, Beruf} und Freizeitverhalten

Im Rahmen der Nachuntersuchung wurde der Patient sowohl zur subjektiven Einschätzung des Operationsergebnisses als auch zur Belastungsfähigkeit und Gehstrecke befragt.

Nach Versorgung mit der IlizarovTechnik war eine deutlich höhere Belastungsfähigkeit zu verzeichnen. $73 \%$ ( $n=$ 8) der Patienten gaben keine Schmerzen unter Belastung oder Schmerzen nur nach größeren Belastungen an. $73 \%(n=$ 8) konnten unverändert ihren gewohnten Freizeitaktivitäten nachgehen und alle 5 zuvor berufstätigen Patienten konnten ohne Probleme in ihren Beruf reintegriert werden.

Nach Versorgung mit konventionellen Operationsverfahren äußerten im Rahmen der Nachuntersuchung 26\% der Patienten $(n=10)$ Ruheschmerzen oder Schmerzen nach geringer Belastung. Nur bei $41 \%$ der Fälle $(n=16)$ trat keine Änderung des Freizeitverhaltens auf. Von den 28 berufstätigen Patienten konnten lediglich $75 \%(n=21)$ ihren Beruf wieder ausüben, $19 \%(n=5)$ wurden umgeschult, und $7 \%(n=2)$ wurden arbeitslos.

Entsprechend wurde das Operationsergebnis von $82 \%(n=9)$ der Patienten der Ilizarov-Gruppe und lediglich von $51 \%(n=20)$ der Patienten der Gruppe II als sehr gut oder gut eingestuft.

\section{Maryland-Foot-Score}

Bei der Evaluierung der Daten anhand des Maryland-Foot-Scores erreichten

- in der Ilizarov-Gruppe (Gruppe I) $82 \%(n=9)$ und

- in der Gruppe II nach Versorgung mit konventionellen Operationsverfahren $69 \%(n=27)$ ein sehr gutes oder gutes Score-Ergebnis.

Ein unbefriedigendes Score-Ergebnis von unter 50 Punkten wurde in der Ilizarov-Gruppe in keinem Fall und in Gruppe II bei 10\% $(n=4)$ beobachtet. Ein signifikanter Unterschied bestand nicht $(p=0,283, p=0,357)$.

Die Evaluierung aller $\mathrm{C}_{2}$ - und $\mathrm{C}_{3}$ Frakturen anhand des Maryland-FootScores demonstriert anschaulich die Effizienz der Ilizarov-Methode für die Versorgung dieser prognostisch ungünstig zu wertenden, komplexen Frakturmorphologie im Vergleich zu konventionellen Operationsverfahren. Ein sehr gutes oder gutes Ergebnis wurde bei $\mathrm{C}_{2}$ - und $\mathrm{C}_{3}$-Frakturen signifikant häufiger mit $87,5 \%$ ( $7 / 8)$ in der Ilizarov-Gruppe als in Gruppe II mit $38 \%$ (5/13) erreicht $(p=0,037)$ (Abb. 7). Bei 1 Patienten der Ilizarov-Gruppe und bei 62\% (8/13) der Patienten nach konventioneller Versorgung war das Score-Ergebnis als befriedigend oder schlecht einzustufen. Die durchschnittlich erreichten Score-Ergebnisse für $\mathrm{C}_{2}$ - und $\mathrm{C}_{3}$-Frakturen von 83 Punkten in der Ilizarov-Gruppe und 68 Punkten nach Therapie mit konventionellen Operationsverfahren unterschieden sich signifikant $(p=0,046)$.

\section{Diskussion}

Hochrasanzverletzungen des Pilon tibiale sind gekennzeichnet durch

- eine komplexe Frakturmorphologie,

- einen schweren Weichteilschaden und

- einen erheblichen Knorpelschaden $[5,12,19,26]$.

Da die Frakturheilung maßgeblich von der Vitalität des ohnehin problematischen Weichteilmantels im Bereich der
Abb. 7 Maryland-Foot-Score der C2- und C3-Frakturen. Ein sehr gutes oder gutes Ergebnis wurde bei C2und C3-Frakturen signifikant häufiger mit $87,5 \%$ in der llizarov-Gruppe als in Gruppe II mit 38\% erreicht $(p=0,037)$. Das durchschnittlich erreichte Score-Ergebnis für C2- und C3-Frakturen unterschied sich ebenfalls signifikant $(p=0,046)$

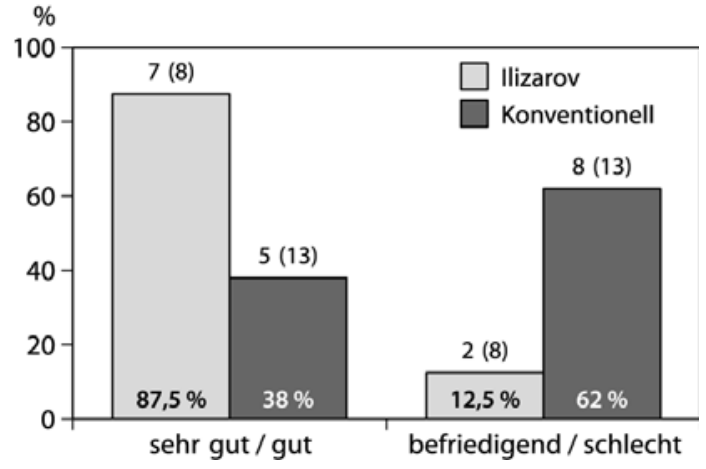


distalen Tibia und dem Erhalt der Vaskularität im Bereich der Frakturzone abhängig ist, wird die Frakturheilung mit Zunahme des traumatischen und auch operationstechnisch bedingten Weichteilschadens entsprechend negativ beeinflusst $[9,19]$. Es überrascht daher nicht, dass mit Zunahme der Frakturkomplexität und der Schwere des Weichteilschadens nachweislich die perioperative Komplikations-, Osteitis- und Pseudarthroseninzidenz ansteigen, schwere Arthroseformen häufiger beobachtet werden und das Endergebnis nachhaltig negativ beeinflusst wird [12, $19,20,21,26,35]$. So wird nach Rommens et al. [25, 26] das Endresultat maßgeblich durch die Schwere des primär bestehenden Weichteilschadens bestimmt, der bei komplexen Pilon-tibiale-Frakturen mit schwerem Weichteilschaden in keinem der Fälle ein gutes Endergebnis erreichen konnte [26].

Neben Fakturmorphologie und Weichteilschaden wird das Ausheilungsergebnis zudem durch eine stufenlose anatomische Rekonstruktion der Gelenkfläche sowie durch das Ausmaß des traumatisch bedingten Knorpelschadens beeinflusst $[3,7,9,12,19,20,21,23$, $26,33,35$ ].

Der traumatisch bedingte Knorpelschaden ist eine zum gegenwärtigen Zeitpunkt kaum zu beeinflussende Determinante. Da autologe Knorpelzelltransplantationen im Bereich des oberen Sprunggelenks zurzeit überzeugende Ergebnisse vermissen lassen [34], kann lediglich durch eine frühfunktionelle Übungsbehandlung mit Verbesserung der nutritiven Situation der Verlauf der Knorpelschädigung positiv beeinflusst werden [36].

Ziele einer jeden operativen Therapie von Pilon-tibiale-Frakturen müssen daher

- eine stufenlose Rekonstruktion der Gelenkfläche unter Vermeidung einer extensiven Weichteildissektion und

- eine zuverlässige, stabile Retention, die eine frühfunktionelle Nachbehandlung gestattet,

beinhalten. Eine anatomische Reposition und zuverlässige Retention sind bei komplexer Frakturmorphologie, unabhängig von der Wahl des Osteosyntheseverfahrens, schwierig und in manchen
Fällen sogar unmöglich $[12,37]$. Insbesondere unter dem Aspekt des traumatischen und operativ bedingten Weichteilschadens werden die Vorgehensweise und die Implantatwahl bei komplexen Pilon-tibiale-Frakturen kontrovers diskutiert. Entsprechend dem mechanisch geprägten Verständnis der 6oer und 7oer Jahre propagieren einige Autoren nach wie vor eine Plattenosteosynthese [4, 12, 21, 33], während andere Autoren, im Zeitalter der biologischen Osteosynthese, bei Pilon-tibiale-Frakturen mit schwerem Weichteilschaden die Minimalosteosynthese favorisieren $[1,5,9,16$, $17,18,19,35,36,37]$.

\section{Plattenosteosynthese}

Teeny u. Wiss [35] berichteten nach Versorgung von komplexen Pilon-tibialeFrakturen durch Plattenosteosynthese über eine Komplikationsrate von $70 \%$ und eine Infektionsrate von $37 \%$. In einer randomisierten Studie von Wyrsch et al. [38] wurden nach interner Fixation mit Plattenosteosynthese eine Infektionsrate von $28 \%$, Wundheilungsstörungen bei 33\% und eine Amputationsrate in $16 \%$ der Fälle und nach externen Fixationsverfahren eine Infektionsrate von $5 \%$, Wundheilungsstörungen in 5\% und in keinem Fall eine Amputation verzeichnet. Andere Autoren publizierten nach primärer Plattenosteosynthese bei High-energy-Verletzungen über eine Infektionsrate von bis $\mathrm{zu} 50 \%[9,10$, 13, 32]. Aufgrund dieser hohen Infektionsinzidenz wird in jüngster Zeit zunehmend ein zweizeitiges Verfahren mit primärer Minimalosteosynthese und sekundärer Plattenosteosynthese nach Konsolidierung der Weichteilverhältnisse propagiert $[4,21,26,33]$.

Obwohl Sirkin u. Sanders [33] bei 46 nachuntersuchten Patienten mit C-Verletzungen durch ein protokolliertes zweizeitiges Vorgehen die Infektionsrate (Osteitisrate $7 \%$, Wundheilungsstörungen $15 \%$ ) deutlich senken konnten, muss die Wertigkeit der sekundären Plattenosteosynthese nach den Ergebnissen von Breitfuß et al. [9], die eine Osteitisrate von $6 \%$ nach Minimalosteosynthese, $33 \%$ nach primärer und $23 \%$ nach sekundärer Plattenosteosynthese fanden, weiter kritisch hinterfragt werden.

Nachteilig erscheint auch, dass die Rekonstruktion der tibialen Gelenkfläche beim zweizeitigen Vorgehen häufig erst nach Konsolidierung der Weichteile in einer Phase erfolgen kann, in der die bereits eingesetzte Kallusbildung die ohnehin schwierige anatomische Reposition noch problematischer gestaltet. So konnte beim zweizeitigen Vorgehen die sekundäre Plattenosteosynthese bei Patterson et al. [21] erst nach durchschnittlich 24 Tagen (15-49 Tagen) und bei Bastian u. Tscherne [4] erst nach durchschnittlich 17 Tagen (5-33 Tagen) erfolgen.

Kritisch stimmt auch die Tatsache, dass nach offener Retention mit Plattenosteosynthese Arthrosen häufiger und mit schwererem Verlauf gesehen werden $[11,13,19]$. So beobachteten Nast-Kolb et al. [19] nach offener Reposition und Plattenosteosynthese trotz leichteren Frakturformen wesentlich häufiger schwere und sehr schwere arthrotische Veränderungen (43\%) als nach Minimalosteosynthese (18\%). Ähnliche Beobachtungen beschrieben Höntzsch et al. [13], die eine Arthroserate von $39 \%$ nach Tibiaplattenosteosynthese und von $12,5 \%$ nach externen Stabilisierungsverfahren ermittelten. Schließlich berichtete Heim [11] in der Gruppe der C3.3-Frakturen von überdurchschnittlich häufig vorkommenden schweren Arthrosen bei der Verwendung langer gerader Platten und räumte ein, dass die Wahl eines solchen großvolumigen Implantats einen ungünstigen Einfluss ausübe.

Die erhöhte Infektions- und Arthroserate nach Plattenosteosynthese sind weitgehend auf die, bei Verwendung eines großvolumigen Implantats, iatrogene Weichteildissektion mit Denudierung einzelner Knochenfragmente zurückzuführen $[4,5,19,23,33]$.

So konnten Resch et al. [24] zeigen, dass verbliebene intraartikuläre Stufen im Tibiaplafond nach offener Reposition eine deutlich schwerwiegendere Arthroseentwicklung bedingten als vergleichbare Gelenkstufen konservativ behandelter Fälle. Folglich ist nach Resch et al. [24] die durch den operativen Zugangsweg bedingte iatrogene Weichteildissektion mit Devaskularisierung von Knochenanteilen für eine verstärkte Arthroseentwicklung verantwortlich.

Selbst für Protektionisten der Tibiaplattenosteosynthese wie Helfet et al. [12] ist der Erhalt der Vitalität von Knochen und Weichteilgewebe der bedeutendste Faktor für ein erfolgreiches operatives Vorgehen. 


\section{Minimalosteosynthesen}

Minimalosteosynthesen in Form von Fixateur-externe-Verfahren mit oder ohne limitierter interner Fixation verwirklichen diese Forderung nach größtmöglicher Weichteilschonung und konnten folglich die Infektions- und Komplikationsrate deutlich senken $[1,5,16,17,18$, $19,35,36,37]$. Als nachteilig erwiesen sich, bei nur alleiniger Anwendung von externen Stabilisierungsverfahren, die nur unzureichende, weil indirekte Reposition von impaktierten artikulären Fragmenten über Ligamentotaxis $[6,12$, $16,21]$ und die meist notwendige gelenküberbrückende Montage, die eine frühfunktionelle Übungsbehandlung verhindert [1]. Eine minimalinvasive perkutane oder arthroskopisch gestützte, alleinige Schraubenosteosynthese ist bei vorliegenden Spaltbrüchen (AOKlassifikation B1-, C1-Frakturen) als weichteilschonende Maßnahme sinnvoll [19], jedoch beim Vorliegen einer metaphysären Trümmerzone (AO-Klassifikation, B3-, C2- und C3-Frakturen) nicht in der Lage, die Kräfte zwischen Epi- und Metaphyse zu neutralisieren [26]. Als Alternative zur Plattenosteosynthese wird daher bei komplexen Pilonfrakturen mit schwerem Weichteilschaden die Kombination eines Fixateur-externe-Verfahrens mit einer limitierten offenen Reposition und Retention durch eine Schraubenosteosynthese empfohlen [16].

\section{Kombination eines Fixateur-externe- Verfahrens mit einer limitierten offenen Reposition und Retention durch eine Schraubenosteosynthese}

Die von uns angewendete Ilizarov-Technik in Kombination mit einer arthroskopisch gestützten oder limitiert offenen Reposition und Retention der Tibiaplafondfragmente durch Schraubenosteosynthese vermeidet die Nachteile der konventionellen Fixateur-externe-Verfahren mit ihrer begrenzten Belastungsfähigkeit sowie der langen, notwendigen gelenküberbrückenden Konsolidierungszeit [31]. Eine nicht gelenküberbrückende Ilizarov-Montage erlaubt, im Gegensatz zu den meisten konventionellen Fixateur-externe-Verfahren, eine frühfunktionelle Übungsbehandlung des OSG, wodurch die nutritive Situation von Weichteilen, Knochen und Knorpel begünstigt wird und Kapselschrump- fung, Muskelatrophie und -kontrakturen minimiert werden.

Wesentliches biomechanisches Merkmal des Ilizarov-Systems ist das nicht lineare, axiale Steifigkeitsverhalten [15, 30]. Dieses dynamische Prinzip lässt, im Gegensatz zu rigiden konventionellen Fixateur-externe-Verfahren oder einer statischen Plattenosteosynthese, bei geringen axialen Belastungen dosierte axiale Bewegungsauslenkungen zu und fördert dadurch die reparativen Prozesse der Frakturheilung, während unter zunehmender Belastung das Steifigkeitsverhalten ansteigt und ein Schutz der reparativen Knochenprozesse vor Gewebeüberdehnung bzw. zu großer Bewegungsauslenkung im Frakturspalt gewährleistet wird. Dieses dynamische Prinzip, welches dem heutigen Verständnis der reparativen Knochenbruchheilung am nächsten kommt, erklärt, warum Pseudarthrosen oder eine verzögerte Knochenbruchheilung unter der Therapie mit dem Ilizarov-System trotz des hohen Anteils an C2- und $\mathrm{C}_{3}$-Frakturen in dieser Studie nicht beobachtet wurden. Pugh et al. [22] zeigten in einer vergleichenden Studie „Ilizarov-HybridFixateur vs. konventionellen Fixateur externe (Orthofix) mit limitierter interner Osteosynthese vs. offene Reposition mit Plattenosteosynthese“, dass unter der Therapie mit dem Ilizarov-Hybrid-Fixateur als einziges Osteosyntheseverfahren keine Pseudarthrose auftrat und bestätigten unsere sowie die Ergebnisse anderer Studiengruppen $[1,16,17,18,36]$. Im Gegensatz zu diesen einheitlich positiven Erfahrungen berichtete Anglen [2] als einziger Autor über eine hohe Pseudarthrosenrate von $24 \%$ nach Ilizarov-Hybrid-Montage. Gründe hierfür liegen u. E. in einer zu geringen Rate an Spongiosaplastiken (lediglich 15,6\% der Fälle) trotz eines hohen Anteils an komplexen Trümmerbrüchen mit diaphysärer Beteiligung und einer zu langen Teilbelastungsphase bei einer durchschnittlich erst nach 147 Tagen erreichten Vollbelastung.

Ein weiterer Vorteil des IlizarovSystems liegt in der hohen primären Stabilität des dreidimensionalen Systems $[15,30,31]$, wodurch eine frühe Belastungsfähigkeit bis zur Vollbelastung bei gleichzeitiger Freigabe des oberen Sprunggelenks gewährleistet wird. Möglicherweise sind dadurch die bessere Beweglichkeit und die früher erreichte Vollbe- lastung in der Ilizarov-Gruppe im Vergleich zur Gruppe II erklärbar.

Aufgetretene Fehlstellungen in der Ilizarov-Gruppe waren weniger Ausdruck einer mangelnden Stabilität als das Problem einer notwendigen vorzeitigen Entfernung des Systems bei aufgetretenem Pininfekt.

Durch die hohe Stabilität des Ilizarov-Systems kann auf eine mediale $\mathrm{Ab}$ stützung durch Plattenosteosynthese verzichtet werden. Die damit gewonnene Gewebeschonung durch Vermeidung der zugangsbedingten Weichteildissektion mit Denudierung von Knochenfragmenten charakterisiert die IlizarovTechnik als „biologische“ Osteosynthese. Dies wird durch die Beobachtung unterstrichen, dass trotz des Vorliegens von schweren und schwersten Weichteilschäden und eines hohen Anteils an $\mathrm{C}_{2}$ - und $\mathrm{C}_{3}$-Frakturen in der IlizarovGruppe keine Osteitis auftrat. Andere Autoren beobachteten nach Therapie mit dem Ilizarov-System ebenfalls keine Osteitis $[1,16,17,18]$, während Tornetta et al. [36] eine Osteitisrate von 3,8\% fanden.

Der Nachteil des Ilizarov-Systems besteht in seiner methodenabhängigen hohen Pininfektinzidenz von 38-69\% [1, $16,17]$. Als typische Komplikation des Ilizarov-Systems verzeichneten wir eine Pininfektionsrate von $45 \%$ mit revisionspflichtigen Befunden in $18 \%$ der Fälle. Alle Pininfekte in der hier vorliegenden Untersuchung und auch in den zitierten Studien heilten ohne Folgen und ohne notwendigen Verfahrenswechsel aus.

Schwere Arthroseformen wurden in dieser Studie in beiden Gruppen mit einer Häufigkeit von jeweils $18 \%$ beobachtet. Die Ausbildung einer posttraumatischen Arthrose hängt maßgeblich vom Zerstörungsgrad der Gelenkfläche, dem vorliegenden Weichteilschaden, dem Auftreten von tiefen Infektionen und der iatrogenen Weichteildissektion ab $[4,5,8,19,20,23,24,26,32]$. Ein Vergleich beider Gruppen ist wegen des statistisch signifikant höheren Anteils an $\mathrm{C}_{2}$ - und $\mathrm{C}_{3}$-Frakturen und schweren Weichteilschäden in der Ilizarov-Gruppe nicht möglich.

Nast-Kolb et al. [19] berichteten nach Versorgung mit Plattenosteosynthese über schwere Arthroseformen in 45\% und bei isolierter Auswertung der C-Frakturen in $75 \%$ der Fälle. Die im 
Vergleich dazu von uns nachgewiesene wesentlich niedrigere Arthroserate nach Therapie mit dem Ilizarov-System ist in Anlehnung an die Ausführungen von Resch et. al. [24] am ehesten auf die geringere iatrogene Weichteildissektion beim Ilizarov-Prozedere zurückzuführen.

Die Arthrodesenhäufigkeit nach Pilon-tibiale-Frakturen schwankt in der Literatur zwischen $5 \%$ und über 30\% [4, $8,32,35$ ]. Bourne et al. [8] publizierten eine Arthrodesenrate nach Tibiaplattenosteosynthese von $32 \%$. In unserem Patientengut war eine Arthrodese in der Ilizarov-Gruppe in keinem Fall und nach Therapie mit konventionellen Operationsverfahren in $8 \%$ der Fälle notwendig. Studien anderer Autoren bestätigten unsere positiven Erfahrungen mit dem Ilizarov-System und beschrieben eine Arthrodesenrate unter Therapie mit dem Ilizarov-System von $0-5 \%$ [1, $16,36]$.

Trotz der ermutigenden Berichte vieler Studiengruppen $[1,16,17,22,36]$ mit exzellenten bis guten Ergebnissen in 70-79\% der Fälle nach Therapie mit dem Ilizarov-System berichtete Anglen [2] von schlechteren klinischen Ergebnissen, höheren Komplikationsraten, höheren Pseudarthrosenraten und längeren Rehabilitationsphasen nach Therapie mit dem Ilizarov-Hybrid-System im Vergleich zur Plattenosteosynthese. Die schlechteren Ergebnisse in der Ilizarov-Hybrid-System-Gruppe traten nach Angaben des Autors aufgrund einer gewissen Patientenselektion nicht unerwartet auf, da Frakturen mit schwerstem Weichteilschaden und hochgradige Trümmerfrakturen sowie Frakturen mit „extensiver“ Beteiligung der Diaphyse fast ausnahmslos mit der Ilizarov-Hybrid-Technik behandelt worden waren. Daher bestand in der Ilizarov-HybridGruppe ein signifikant höherer Anteil an offenen Frakturen, Frakturen mit höhergradig geschlossenem Weichteilschaden und komplexen Frakturmorphologien (C-Frakturen) als in der Vergleichsgruppe, die mit Plattenosteosynthese behandelt wurde. Des Weiteren wurden die tendenziell schlechteren Ergebnisse der Ilizarov-Hybrid-Gruppe durch einen höheren Anteil an polytraumatisierten Patienten und ipsilateralen Extremitätenverletzungen nachhaltig beeinflusst. Zudem war die Anzahl an unbefriedigenden Repositionsergebnissen in der Ilizarov-Gruppe
(20\%) doppelt so hoch wie in der Vergleichsgruppe mit Plattenosteosynthese (10\%). Aufgrund dieser selektionsbedingten Gruppenunterschiede sind das schlechtere klinische Ergebnis und die längere Rehabilitationsphase in der Ilizarov-Hybrid-Gruppe erklärbar.

Im Gegensatz zu der Studie von Anglen [2] konnten in der hier vorliegenden Studie die mit dem Ilizarov-System behandelten Patienten schneller frühfunktionell nachbehandelt werden, erreichten rascher eine Vollbelastung und gaben tendenziell eine geringere Einschränkung der Gehstrecke sowie weniger Schmerzen unter Belastung an als Patienten, die mit konventionellen Operationsverfahren behandelt wurden.

Auch wenn in der Ilizarov-Gruppe ein signifikant höherer Anteil an C2und $\mathrm{C}_{3}$-Frakturen, an schweren Weichteilschäden, an älteren Patienten und Vorschäden im Bereich des OSG zu verzeichnen waren, wurden die Ausheilungsergebnisse in Gruppe II durch einen signifikant höheren Anteil an konkomitierenden Talus- und Kalkaneusfrakturen sowie polytraumatisierten und mehrfach verletzten Patienten nachhaltig beeinflusst.

Dennoch lässt sich die Effizienz des Ilizarov-Systems in der Therapie von $\mathrm{C}_{2}$ und $\mathrm{C}_{3}$-Frakturen bei einem signifikant besseren Score-Ergebnis (MarylandFoot-Score) im Vergleich zur Gruppe mit konventionellen Operationsverfahren nicht bestreiten. Ein sehr gutes oder gutes Score-Ergebnis von $87 \%$ der Patienten nach Versorgung mit der IlizarovTechnik und von 38\% der Patienten mit konventionellen Operationsverfahren bei alleiniger Auswertung der $\mathrm{C}_{2}$ - und $\mathrm{C}_{3}$-Frakturen unterstreichen dies nachhaltig. Unsere Resultate mit der IlizarovTechnik zeigten eine niedrigere Osteitis, Arthrose- und Pseudarthrosenrate und ein besseres klinisches Ergebnis als die meisten anderen Studien mit vergleichbaren Patientenkollektiven.

\section{Schlussfolgerung}

Das Ilizarov-System besticht durch eine kaum zu überbietende Gewebeschonung und eine hohe primäre Belastungsfähigkeit, die eine frühfunktionelle Nachbehandlung erlaubt.

Mit der Ilizarov-Technik wurden trotz eines statistisch signifikant höheren Anteils an 43-C2/C3-Frakturen und schweren Weichteilschäden bessere Ergebnisse erreicht als in einer Vergleichsgruppe mit konventionellen Operationsverfahren.

Die Ilizarov-Technik in Kombination mit einer minimal traumatisierenden, internen Osteosynthese ist ein effizientes Verfahren zur Therapie von komplexen Pilon-tibiale-Frakturen mit schwerem Weichteilschaden.

\section{Literatur}

1. Aktuglu K, Özsoy MH, Yensel U (1998) Treatment of displaced pylon fractures with circular external fixators of llizarov. Foot Ankle Int 19: 208-216

2. Anglen JO (1999) Early outcome of hybrid external fixation for fracture of the distal tibia. J Orthop Trauma 13:2

3. Babis GC, Vayanos ED, Papaioannou N, Pantazopoulos Th (1997) Results of surgical treatment of tibial plafond fractures. Clin Orthop 341: 99-105

4. Bastian L, Blauth $\mathrm{M}$, Thermann $\mathrm{H}$, Tscherne $\mathrm{H}$ (1995) Verschiedene Therapiekonzepte bei schweren Frakturen des Pilon tibiale (Typ-CVerletzungen). Unfallchirurg 98: 551-558

5. Bone L, Stegemann Ph, McNamara K, Seibel R (1993) External fixation of severely comminuted and open tibial pilon fractures. Clin Orthop 292: 101-107

6. Borrelli Jr J, Catalano L (1999) Open reduction and internal fixation of pilon fractures.J Orthop Trauma 13:573-581

7. Borrelli J Jr, Torzilli PA, Grigiene R, Helfet DL (1997) Effect of impact loading on articular cartilage: development of an intra-articular fracture model. J Orthop Trauma 11:319-326

8. Bourne RB, Rorabeck CH, MacNab I (1983) Intraarticular fractures of the distal tibia: the piIon fracture.J Trauma 23: 591

9. Breitfuss $\mathrm{H}$, Muhr $\mathrm{G}$, Neumann K, Korthaus C (1988) Prognose und Therapie geschlossener, distaler intraartikulärer Unterschenkelbrüche. Unfallchirurg 91: 557-564

10. Dillin L, Slabaugh P (1986) Delayed wound healing, infection, and nonunion following open reduction and internal fixation of tibial plafond fractures.J Trauma 26: 1116

11. Heim U (1991) Die Pilon-tibial-Fraktur: Klassifikation, Operationstechnik, Ergebnisse. Springer, Berlin Heidelberg New York, S 288

12. Helfet DL, Koval K, Pappas J, Sanders RW, DiPasquale T (1994) Intraarticular "pilon“ fracture of the tibia. Clin Orthop 298: 221-228

13. Höntzsch D, Karnatz N, Jansen T (1990) Einoder zweizeitige (mit Fixateur externe) Versorgung der schweren Pilon-tibial-Fraktur. Aktuelle Traumatol 20: 199-204

14. Jergesen $F(1959)$ Fractures of the ankle. Am J Surg 98: 136-145

15. Jürgens C, Schmidt HGK, Fink B (1992) Der Ilisarow-Ringfixateur und seine technische Anwendung. Unfallchirurg 95:529-533 


\section{Unterschenkel- und obere Sprunggelenksfrakturen}

16. Kim H-S, Jahng J-S, Kim S-S, Chun Ch-H, Han H-J (1997) Treatment of tibial pilon fractures using ring fixators and arthroscopy. Clin Orthop 334: $244-250$

17. Mc Donald MG, Burgess RC, Bolano LE, Nicholls PJ (1996) Ilizarov treatment of pilon fractures. Clin Orthop 325: 232-238

18. Murphy CP, D'Ambrosia R, Dabezies EJ (1991) The small pin circular fixator for distal tibial pilon fractures with soft tissue compromise. Orthopedics 19: 283-290

19. Nast-Kolb D, Betz A, Rödel C, Schweiberer L (1993) Die Minimalosteosynthese der Pilontibial-Fraktur. Unfallchirurg 96: 517-523

20. Ovadia DN, Beals RK (1986) Fractures of the tibial plafond.J Bone Joint Surg Am 68A: 543-551

21. Patterson MJ, Cole JD (1999) Two-staged delayed open reduction and internal fixation of severe pilon fractures.J Orthop Trauma 13: 85-91

22. Pugh KJ, Wolinsky PhR, Mc Andrew MP, Johnson KD (1999) Tibial pilon fractures: a comparison of treatment methods.J Trauma 47: 937941

23. Resch H, Benedetto KP, Pechlaner S (1986) Die Entwicklung der posttraumatischen Arthrose nach Pilon-Tibialfrakturen. Unfallchirurg 89: 8-15
24. Resch H, Pechlaner S, Benedetto KP (1986) Spätergebnisse nach konservativer und operativer Behandlung von Pilon Tibial-Frakturen. Aktuelle Traumatol 16: 117-123

25. Rommens PM, Broos PL (1992) The significance of soft tissue trauma for fracture healing. A prospective study on 70 tibial shaft fractures following primary treatment with the monofixateur.Unfallchirurg 95: 133

26. Rommens PM, Claes P, De Boodt P, Stappaerts KH, Broos PL (1994) Therapeutisches Vorgehen und Langzeitergebnisse bei der Pilonfraktur in Abhängigkeit vom primären Weichteilschaden. Unfallchirurg 97: 39-46

27. Ruedi T, Allgöwer M (1969) Fractures of the lower end of the tibia into the ankle joint. Injury 1: 92

28. Ruedi T, Allgöwer M (1978) Spätresultate nach operativer Behandlung der Gelenkbrüche am distalen Tibiaende ( sog.Pilonfrakturen). Unfallheilkunde 81:319-323

29. Sanders $R$, Fortin $P$, DiPasquale Th, Walling $A$ (1993) Operative treatment in 120 displaced intraarticular calcaneal fracture. Clin Orthop 290: 87-95

30. Schneider E, Sasse S, Schmidt HGK, Schümann U (1992) Zur Biomechanik des Ringfixateurs Beiträge einzelner Strukturelemente. Unfallchirurg 95: 580-587

31. Schultz J-H, Wolter D, Ortel G, Fink B (1992) Die Frakturbehandlung im Unterschenkelbereich. Unfallchirurg 95: 537-540
32. Schweiberer L, Betz A, Nast-Kolb D, Bischoff B (1987) Spezielle Behandlungsmethodik am distalen Unterschenkel und bei Pilonfrakturen. Unfallchirurgie 90: 253-259

33. Sirkin $M$, Sanders R, DiPasquale Th, Jr Herscovici D (1999) A staged protocol for soft tissue management in the treatment of complex pilon fractures.J Orthop Trauma 13: 78-84

34. Steinwachs MR, Erggelet C, Lahm A, GuhlkeSteinwachs U (1999) Klinische und zellbiologische Aspekte der autologen Chondrozytenimplantation. Unfallchirurg 102: 855-860

35. Teeny StM, Wiss AD (1993) Open reduction and internal fixation of tibial plafond fractures. Clin Orthop 292: 108-117

36. Tornetta III P, Weiner L, Bergman M, Watnik N, Steuer J, Kelley M, Yang E (1993) Pilon fractures: treatment with combined internal and external fixation.J Orthop Trauma 7: 489-496

37. Treadwell JR, Fallat LM (1994) Dynamic unilateral distraction fixation: surgical management of tibial pilon fractures.J Foot Ankle Surg 33: 438-442

38. Wyrsch B, McFerran MA, Mc Andrews M, Limbird TJ, Harper MC, Johnson KD, Schwartz HS (1996) Operative treatment of fracture of the tibia plafond. A randomized, prospective study. J Bone Joint Surg Am 78: 1646-1657 